\title{
Variations in Copepod Proteome and Respiration Rate in Association with Diel Vertical Migration and Circadian Cycle
}

\author{
AMY E. MAAS ${ }^{1, *}$, LEOCADIO BLANCO-BERCIAL $^{1}$, ALI LO $^{2}$, ANN M. TARRANT $^{3}$, \\ AND EMMA TIMMINS-SCHIFFMAN \\ ${ }^{1}$ Bermuda Institute of Ocean Sciences, 17 Biological Station, St. George's GE01, Bermuda; ${ }^{2}$ Department \\ of Genome Sciences, University of Washington, Seattle, Washington 98195; and ${ }^{3}$ Biology Department, \\ Woods Hole Oceanographic Institution, 266 Woods Hole Road, Woods Hole, Massachusetts 02543
}

\begin{abstract}
The diel vertical migration of zooplankton is a process during which individuals spend the night in surface waters and retreat to depth during the daytime, with substantial implications for carbon transport and the ecology of midwater ecosystems. The physiological consequences of this daily pattern have, however, been poorly studied beyond investigations of speed and the energetic cost of swimming. Many other processes are likely influenced, such as fuel use, energetic tradeoffs, underlying diel (circadian) rhythms, and antioxidant responses. Using a new reference transcriptome, proteomic analyses were applied to compare the physiological state of a migratory copepod, Pleuromamma xiphias, immediately after arriving to the surface at night and six hours later. Oxygen consumption was monitored semi-continuously to explore underlying cyclical patterns in metabolic rate under darkdark conditions. The proteomic analysis suggests a distinct shift in physiology that reflects migratory exertion and changes in metabolism. These proteomic analyses are supported by the
\end{abstract}

Received 22 January 2018; Accepted 11 June 2018; Published online August 16, 2018.

* To whom correspondence should be addressed. E-mail: amy.maas@bios .edu.

Abbreviations: ACN, acetonitrile; ANOSIM, analysis of similarity; BATS, Bermuda Atlantic Time Series; BUSCO, Benchmarking Universal Single-Copy Orthologs; DM, dry mass; DVM, diel vertical migration; FFT-NLLS, fast Fourier transform non-linear least squares; GO, gene ontology; MESA, maximum entropy spectral analysis; NAD, nicotinamide adenine dinucleotide; $\mathrm{NAD}+$, oxidized nicotinamide adenine dinucleotide; $\mathrm{NADH}$, reduced nicotinamide adenine dinucleotide; NMDS, non-metric multidimensional scaling; nr, non-redundant database; NSAF, normalized spectral abundance factor; RT, room temperature; TTP, Trans Proteomic Pipeline.

Online enhancements: data files. respiration experiments, which show an underlying cycle in metabolic rate, with a peak at dawn. This project generates molecular tools (transcriptome and proteome) that will allow for more detailed understanding of the underlying physiological processes that influence and are influenced by diel vertical migration. Further, these studies suggest that $P$. xiphias is a tractable model for continuing investigations of circadian and diel vertical migration influences on plankton physiology. Previous studies did not account for this cyclic pattern of respiration and may therefore have unrepresented respiratory carbon fluxes from copepods by about $24 \%$.

\section{Introduction}

One of the greatest cyclical patterns in the pelagic ecosystem is the daily migration of various zooplankton and fish to depth, a process referred to as diel vertical migration (DVM). DVM is typified by the presence of transient species in the warm, oxygenated, photic zone during the night, followed by a movement of individuals into colder, deeper water during the day (although variations, including reverse migrations, exist; Heywood, 1996; Hays, 2003). These daily migrations are thought to be energetically expensive, with individuals smaller than $1 \mathrm{~cm}$ traveling hundreds of meters per day. DVM speed has been estimated, via calculations of the observed amplitude and duration of DVM for copepods and euphausiids, to range between 10 and $55 \mathrm{~mm} \mathrm{~s}^{-1}$ (Wiebe et al., 1992; Mauchline, 1998). The estimates of the physiological costs of such profound exertion, based on force transducer and video analyses, range between $13 \%$ and $120 \%$ of copepod basal metabolic rate (reviewed in Mauchline, 1998). Despite the costs, the process is believed to provide a number of advantages to migratory or- 
ganisms, including niche partitioning, metabolic advantage due to colder temperatures at depth, retreat from light or high temperatures, and, most importantly, predator avoidance (Hays, 2003; Antezana, 2009).

Globally, the DVM of the zooplankton community is estimated to be responsible for $15 \%-40 \%$ of the total organic carbon export from the surface to the mesopelagic (Steinberg et al., 2000; Bianchi and Mislan, 2016), exceeding in many cases the carbon flux related to passively sinking particles (Kobari et al., 2003, 2008; Steinberg et al., 2008; Steinberg and Landry, 2017). Similar percentages are reported for the nitrogen-related processes (e.g., Dam et al., 1995; Al-Mutairi and Landry, 2001; Schnetzer and Steinberg, 2002b; Steinberg et al., 2002). These estimates are typically made by applying mass-specific and temperature-scaling factors to experimentally calculated oxygen consumption, as well as nitrogen and carbon excretion by individual organisms. These experiments are traditionally done by placing individuals in bottles for long durations (8-24 h), with no mention of the start time of the experiment; and, thus, variations in physiological state that are a consequence of circadian patterns or the DVM process are lost. If there are substantial physiological changes associated with migration or hidden daily cycles in major physiological processes, such as oxygen consumption and ammonia excretion, they may cause errors in the estimations of organismal contributions to biogeochemical flux during daytime at depth. Previous studies have suggested that this may indeed be the case, with the migratory copepods Pleuromamma xiphias and Calanus euxinus showing substantially higher respiration rates late in the evening (22:00) and early in the morning (05:00) relative to the nadir at mid-day (13:00) (Pavlova, 1994). The design of these studies still relied upon noncontinuous respirometry measurements (3-6-h endpoint incubations), making a pinpointed analysis of the variation in physiological response impossible.

To explore the physiological signature of DVM, more finely resolved measurements are required. Furthermore, although it is important to define the energetic cost of swimming, understanding the full physiological implications of migrations requires analysis of more complex mechanisms such as changes in metabolic pathways, endogenous circadian rhythms, and antioxidant activity. These topics have been explored in terrestrial migratory studies (i.e., Gwinner and Helm, 2003; McGuire and Guglielmo, 2009; Skrip and McWilliams, 2016) but have not, to date, received much attention in zooplankton because of the difficulty of organismal sampling, the small size of individuals, and the lack of appropriate molecular tools to adequately quantify changes. Under natural conditions, timing of planktonic DVM is largely cued by daily light-dark cycles, and over time animals become synchronized, or entrained, to this predictable environmental cue. By showing that daily movement patterns can persist in migratory crustaceans in constant darkness, laboratory-based studies have indicated that DVM could be regulated by an endogenous circadian clock (Enright and Hamner, 1967), thus suggesting that there is still much to be learned in this system.

New advances in molecular techniques have recently opened the door for more detailed analyses of these more complex aspects of DVM, and research regarding the influence of circadian patterns on diel and seasonal migrations is underway (Cohen and Forward, 2009; Teschke et al., 2011; Christie et al., 2013; De Pitta et al., 2013). A recent analysis using semi-continuous optical oxygen sensing, characterization of DVM behavior, and expression of core clock genes via quantitative polymerase chain reaction (qPCR) revealed that the copepod Calanus finmarchicus has a true circadian rhythm in metabolism, with a suggested peak in respiration during the early evening (Häfker et al., 2017). This sets the stage for studies that investigate which other processes may also be influenced by both migratory behavior and circadian cycles.

The goal of this project was to determine whether it is possible to observe biogeochemically relevant diel changes in the physiology of a subtropical copepod through organismal (respiration) and molecular (proteomics) methods. To support the latter analysis, we generated a species-specific copepod transcriptome that could then be used to identify copepod proteins and to detail the change in protein abundances that a vertical migratory zooplankton experiences over the nighttime portion of its diel cycle. Pleuromamma xiphias, a well-known vertical migrator at the Bermuda Atlantic Time Series (BATS) site, was chosen for this study because it has previously been shown to have diel patterns in respiration rate that are assumed to be associated with its migratory behavior (Pavlova, 1994). This species is abundant in the Sargasso Sea, and its contributions to nitrogen and carbon flux have previously been characterized using classical bottle sampling methods (Wormuth, 1981; Steinberg et al., 2000, 2002; Schnetzer and Steinberg, 2002b; Teuber et al., 2013), providing greater context for the more detailed physiological assessment enabled by proteomics analysis.

\section{Materials and Methods}

\section{Copepod collection for transcriptomic and proteomic analyses}

Female copepods (Pleuromamma xiphias (Giesbrecht, 1889)) were collected off the coast of Bermuda $\left(31^{\circ} 39.93 \mathrm{~N}\right.$, $\left.64^{\circ} 00.14 \mathrm{~W} ; 31^{\circ} 32.00 \mathrm{~N}, 63^{\circ} 37.02 \mathrm{~W}\right)$ near the BATS site. Sampling was limited to the nighttime because the available net systems precluded capture of healthy organisms from their daytime depth, which is typically $\sim 400-650 \mathrm{~m}$ locally (LB-B and AM, unpubl. data). On April 17, 2016, a 1- $\mathrm{m}^{2}$ square net with a Star-Oddi depth logger (Gardabaer, Iceland) was towed horizontally at 02:45 (local time) to a 120-m depth, then retrieved, with two 6-min pauses to try to target regions below the chlorophyll $a$ maximum (as observed via nearby hydrographic sampling). On April 18 at 20:30 (local time), after a 
19:45 sunset, the same net was deployed to $180 \mathrm{~m}$, towed, and retrieved in an identical fashion. Upon recovery of the net, the cod end was diluted in a 20 -L cooler containing water from the flow-through seawater line. Individuals were then manually captured via a soft pipette and identified under a Leica stereomicroscope (Wetzlar, Germany) to verify species identity and exclude individuals with broken antennae or legs. The copepods were immediately individually flash-frozen in a dry shipper filled with liquid nitrogen. Sorting of all individuals used in subsequent analyses was completed 32 and 34 min after capture (on April 17 and 18, respectively). For simplicity, these samples are subsequently referred to as 02:00 and 20:00.

\section{Respiration experiment}

An additional collection was made offshore from Bermuda $\left(32^{\circ} 20.28 \mathrm{~N}, 64^{\circ} 32.82 \mathrm{~W}\right)$ from a small vessel on May 11, 2017 , between 21:00 and 23:00. Sunrise was 06:23, and sunset was 20:07. Three tows were conducted with a specialized $150-\mu \mathrm{m}$ mesh Reeve net (Sea-Gear, Melbourne, FL) with a large cod end. The net was slowly towed horizontally at a 100-m depth for $15 \mathrm{~min}$ and then retrieved. Animals were gently poured through a 1000- $\mu \mathrm{m}$ mesh sieve and transferred to a cooler containing in situ water. After the third tow, organisms were brought back to the laboratory, and $P$. xiphias were picked out of the coolers. Copepods were placed at low densities ( $<10$ individuals $\left.\mathrm{L}^{-1}\right)$ in $0.2-\mu \mathrm{m}$ filtered in situ water and were maintained in a dark incubator at $20{ }^{\circ} \mathrm{C}$ (temperature at the chlorophyll $a$ maximum) for $1 \mathrm{~h}$ to allow for gut clearance and acclimatization (Bautista and Harris, 1992; Irigoien, 1998). After the acclimatization period, individuals were transferred to fresh $20^{\circ} \mathrm{C} 0.2-\mu \mathrm{m}$ filtered in situ water in $60-\mathrm{mL}$ glass Biological Oxygen Demand (BOD) bottles (Wheaton, Millville, NJ) containing an optically sensitive oxygen sensor (OXFOIL; PyroScience, Aachen, Germany). As a control for bacterial respiration, every fourth chamber was filled with water but was left without a copepod. Chambers were placed in a dark $20^{\circ} \mathrm{C}$ incubator, and the oxygen concentration in each chamber was then measured noninvasively and continuously (every $2 \mathrm{~min}$ ) for $3 \mathrm{~d}$ by using a FireSting optical oxygen meter (PyroScience, Aachen, Germany). At the end of the experiment, individuals were visually inspected to ensure that they were swimming. The respiration rate for each individual $(n=4)$ was corrected for bacterial respiration (on average, $0.06 \mu \mathrm{mol} \mathrm{h}^{-1}$ ) by subtracting the oxygen concentration within the organismal chamber from the control chamber at each time point. Periodicity was visualized by plotting the oxygen consumption rate $\left(\mu \mathrm{mol} \mathrm{O}_{2} \mathrm{~h}^{-1}\right)$ calculated over a 2 -h period. These rates were then binned by $1 \mathrm{~h}$ and plotted versus the midpoint of the calculation period.

To illustrate how the duration or starting point of the respiration experiment may influence calculation of zooplankton respiratory active flux, typical endpoint calculations were made (Oxygen $_{\text {initial }}-$ Oxygen $_{\text {final }} /$ Duration of experiment). Starting values $\left(\right.$ Oxygen $\left._{\text {initial }}\right)$ were chosen as the first measurement (taken at 02:30), then, starting at 06:00 on the first day, every $6 \mathrm{~h}$ for 2 days. Ending values $\left(\mathrm{Oxygen}_{\text {final }}\right.$ ) were taken 6, 12, 18 , or $24 \mathrm{~h}$ after the starting time point, except in the case of the 02:30 measurement, which was calculated using endpoints $3.5,9.5,15.5$, and $21.5 \mathrm{~h}$ after the start of the experiment. Oxygen content during these time periods declined from $100 \%$ to a minimum of $70 \%$. The use of later time points was excluded in case there was an influence of reduced oxygen availability in the chambers on metabolic rate. This is well above the critical oxygen partial pressure ( $p$ crit) typical of most marine invertebrates (Childress and Seibel, 1998) and well above the pcrit measured for the congener Pleuromamma abdominalis at $23{ }^{\circ} \mathrm{C}(<30 \%$ oxygen saturation; Kiko et al., 2016).

Rhythmicity in respiration was evaluated through period analysis as implemented on the BioDare2 web portal (BioDare2, 2016). Linear de-trending was conducted to compensate for the decline in respiration rate over time, which might otherwise obscure detection of rhythmicity (Zielinski et al., 2014). De-trended time series were evaluated using the fast Fourier transform non-linear least squares (FFT-NLLS) algorithm and maximum entropy spectral analysis (MESA) methods. These methods were chosen because they provide error estimates for the period, because they are based on different principles (curve fitting $v s$. stochastic modeling), and because MESA is relatively robust to the presence of baseline trends (Zielinski et al., 2014). Statistical significance of a 24-h period was tested using a waveform-independent nonparametric approach within the R package RAIN, with the "longitudinal" analysis option (Thaben and Westermark, 2014).

\section{Transcriptome generation}

Two males and one female from both the 02:00 and 20:00 capture time points were extracted in two mixed batches of three individuals using the Qiagen universal RNA mini kit (Qiagen, Germantown, MD). A 5- $\mu \mathrm{L}$ aliquot of each extraction was combined (for a total of 6 individuals) and sent to the University of Rochester Genomics Research Center, where a library was constructed using the TruSeq library prep kit (Illumina, San Diego, CA). The cDNA library was sequenced on one lane on an Illumina HiSeq2000 with 125-bp paired-end reads. Raw sequences were quality filtered by the sequencing facility using the program Trimmomatic (ver. 0.32; Bolger et al., 2014). The program was set to eliminate adapter sequences (2:30:10) and to discard low-quality scores using a sliding window (4:20) that removed both trailing and leading sequences (13) and left only sequences with a minimum length of 15 for downstream use. These cleaned sequences were used to assemble a transcriptome using the Trinity software package (ver. 2.1.1; Grabherr et al., 2011) with default parameters $(\mathrm{k}-\mathrm{mer}=25$; cutoff $=200 \mathrm{bp})$. The transcripts within this assembly were clustered with the program CD-HIT EST (ver. 4.6.1; Li and Godzik, 2006) to a similarity of $95 \%$. The 
quality of the transcriptome, read depth, and estimates of expressed genes were explored using the downstream scripts packaged with Trinity and with Benchmarking Universal Single-Copy Orthologs (BUSCO), using default parameters and the metazoan lineage file (ver. 3.0.2; Simão et al., 2015; Waterhouse et al., 2018).

\section{Protein digestion, desalting, and liquid chromatography- tandem mass spectrometry}

To reduce variability within time points, analysis was restricted to adult female copepods ( $n=6$ per time point). These were transported on dry ice to the University of Washington for proteomic analysis. Each copepod was homogenized in $50 \mathrm{mmol} \mathrm{L}{ }^{-1} \mathrm{NH}_{4} \mathrm{HCO}_{3}(100 \mathrm{~mL})$ before $6 \mathrm{~mol} \mathrm{~L}^{-1}$ urea was added. Sonication, digestion, and desalting proceeded as previously described (Timmins-Schiffman et al., 2013), with the additional digestion enzyme LysC (Wako Chemicals, Richmond, VA). Briefly, after sonication and protein quantification, tris- $\mathrm{HCl}\left(1.5 \mathrm{~mol} \mathrm{~L} \mathrm{~L}^{-1}, \mathrm{pH} 8.8\right)$ and tris (2-carboxyethyl)phosphine were added to each sample and incubated for $1 \mathrm{~h}$ at $37^{\circ} \mathrm{C}$. Iodoacetamide was added to alkylate the proteins and incubated for $1 \mathrm{~h}$ in the dark at room temperature (RT). Excess iodoacetamide was absorbed over a 1-h incubation at RT by dithiothreitol. To reduce the number of undigested cleavage sites, samples were preliminarily digested by LysC for $1 \mathrm{~h}$ at RT at a $1: 20$ enzyme : protein ratio. After LysC digestion, $\mathrm{NH}_{4} \mathrm{HCO}_{3}$ and high-performance liquid chromatography-grade methanol were added to each sample to allow trypsin digestion. Trypsin was added in a $1: 20$ ratio and incubated overnight at RT. The next day, samples were evaporated, reconstituted in 5\% acetonitrile $(\mathrm{ACN})$ with $0.1 \%$ trifluoroacetic acid, and desalted using a MacroSpin Column (Nest Group, Southborough, MA), following the manufacturer's instructions. Desalted peptides were dried and reconstituted in $100 \mu \mathrm{L}$ of $5 \% \mathrm{ACN}$ with $0.1 \%$ formic acid.

The mass spectrometry analysis was performed at the University of Washington Proteomics Resource (Seattle, WA). All 12 samples were analyzed on a Q Exactive mass spectrometer (ThermoFisher Scientific, Waltham, MA) under datadependent acquisition mode, injecting equal quantities of peptide $(1 \mu \mathrm{g})$ in triplicate. Peptides were eluted using an acetonitrile gradient $(5 \%-85 \%)$ over the course of $75 \mathrm{~min}$.

\section{Transcriptome and proteome annotation}

The transcriptome assembly was annotated using a BLASTP search against a local UniProt TrEMBL database (UniProt Consortium, 2016), with an e-value cutoff of $1 \mathrm{e}^{-10}$ (Camacho et al., 2009). It was further annotated using a local BLASTX versus the National Center for Biotechnology Information (NCBI; Bethesda, MD) non-redundant (nr) database (ftp://ftp .ncbi.nlm.nih.gov/blast/db/; downloaded from NCBI on January 19,2017 ), with an e-value cutoff of $1 \mathrm{e}^{-5}$. To provide more comprehensive cross annotation with closely related pelagic rustaceans, the assembly was reciprocally blasted using TBLASTX (NCBI) and an e-value cutoff of $1 \mathrm{e}^{-5}$ versus Calanus finmarchicus (GenBank accession no. GBFB01000001.1; Tarrant et al., 2014), Euphausia superba (available from the European Bioinformatics Institute as study PRJEB6147, HACF01000001-HACF01058581; Meyer et al., 2015), and Meganyctiphanes norvegica (GenBank accession no. GETT 00000000.1; Blanco-Bercial and Maas, 2018) transcriptomes. We also performed a BLASTX search with an e-value cutoff of $1 \mathrm{e}^{-5}$ against protein sequences from the well-annotated Drosophila melanogaster using Flybase (http://flybase.org, FB2016_03). Gene families of specific interest (i.e., circadian, molting, metabolic, stress response) in pelagic crustaceans have previously been identified from transcriptomic resources by Meyer et al. (2015) and Christie et al. (2013). These are gene families that are likely to be influenced by diel changes in physiology; therefore, homologs of these genes were identified using the previously described BLASTX and TBLASTX searches of the E. superba assembly or Flybase, respectively.

Peptide identifications and protein inferences for tandem mass spectra for each copepod were found through Comet (2016.01 rev. 2; Eng et al., 2013, 2015) searches and the Trans Proteomic Pipeline (TPP) (Deutsch et al., 2010) against $P$. xiphias predicted protein sequences, translated from the assembled transcriptome. Comet parameters included a concatenated decoy search against fully digested tryptic peptides, allowing for two missed cleavages (for full Comet parameters see Supplemental Data 1; Supplemental Data files 1-4 are available online). A probability cutoff at the peptide level of 0.9 was implemented in the TPP. Abacus was used to coordinate protein inferences across replicates and calculate the normalized spectral abundance factor (NSAF) (Fermin et al., 2011) for proteins with a minimum combined probability of at least 0.9 . Only proteins with at least two spectral counts across all replicates were included in further analysis. The P. xiphias proteome (based on inferred proteins detected through mass spectrometry) was independently annotated with BLASTP against the UniProt TrEMBL database (UniProt Consortium, 2016) with an e-value cutoff of $1 \mathrm{e}^{-10}$. Annotation-associated gene ontology (GO) (du Plessis et al., 2011) annotations were downloaded from UniProt (UniProt Consortium, 2016).

\section{Protein abundance analysis}

Individual copepod protein abundance profiles were compared using non-metric multidimensional scaling (NMDS) and analysis of similarity (ANOSIM) by using the vegan library in R, version 3.3.2 (Oksanen et al., 2016; R Core Team, 2016). NSAF values were $\log (x+1)$ transformed, and a NMDS plot was generated using a Bray-Curtis dissimilarity matrix. Differential abundance of proteins was determined using QSpec (Choi et al., 2008). Spectral counts were summed across technical replicates within biological replicates (cope- 
pods) in the QSpec analysis. A protein was considered differentially abundant if its log-fold change was $>|0.5|$ and its $z$-value was $>|2|$.

Functional enrichment analysis was completed for GO terms associated with differentially abundant proteins. Two protein sets (positive and negative log-fold change) were compared to detect proteins to determine protein functional groups that were overrepresented in the differentially abundant proteins compared to the background. The analysis was performed using an enrichment tool developed in-house and described in Timmins-Schiffman et al. (2017). Briefly, a $P$-value was calculated describing the enrichment of a GO term in a set of tested proteins above what would be expected by chance, given the annotation of the $P$. xiphias proteome. A $P$-value cutoff of 0.01 was used to ascribe statistical significance to GO terms representing biological process, cellular component, or molecular function.

\section{Results}

\section{Respiration experiment}

Continuous monitoring of Pleuromamma xiphias oxygen consumption from 4 separate individuals for $3.5 \mathrm{~d}$ under full dark conditions revealed a consistent $\sim 24$-h oscillation in respiration rate, although the circadian signal was dampened and disappeared over time (Fig. 1A). This may reflect attenuation of the cycle in the absence of an external light cue, artifacts associated with continued monitoring in a small enclosure, or some combination of factors. There was a failure in one of the oxygen meters on the first day (14:39-15:17), leaving a small gap in the measurement of three individuals; however, a cyclical pattern of metabolic rate, with a peak near dawn (local sunrise was 06:23) and a nadir at mid-evening was apparent for all four copepods. Oxygen content in the chambers had fallen from $100 \%$ to $~ 70 \%$ saturation by the beginning of the third day. All individuals were swimming and responsive to touch at the end of the experiment.

Following linear de-trending, 2 complementary methods identified near 24-h periodicity in respiration rate (FFTNLLS: $25.98 \pm 3.83$ SD; MESA: $26.48 \pm 4.84$ SD; FFTNLLS curve fit shown in Fig. 1B). Nonparametric testing of the mean respiration rate indicated a highly significant 24 -h period $\left(P=1.74 \mathrm{e}^{-15}\right)$. Individual profiles also showed significant 24 -h periodicity, and the result was robust in the presence of the overall declining respiration rate.

In order to evaluate the impact of circadian patterns on analyses of respiration rate, comparisons were made among metabolic rates calculated using varying starting times for the first $2.5 \mathrm{~d}$ of the experiment. These analyses reveal diel changes in rate as well as a decrease in oxygen consumption over time that was likely a consequence of typical starvation and captivity stress (Fig. 2A). The discrepancies between values that were calculated over a $24-\mathrm{h}$ interval but with different starting times (which should be the same because they integrate over the full circadian cycle) were used to backcalculate the change in rate due to the captivity effect as

$$
y=3 e^{-11} x^{5.3367},
$$

where $x=$ duration of captivity and $y=$ decline in metabolism attributable to captivity $\left(R^{2}=0.955\right)$. If the metabolic rate calculations are corrected by this simplified approximation of the captivity effect (Fig. 2B), it is obvious that the starting time of the respiration experiment has an important impact on the calculation of metabolic rate in the first $6 \mathrm{~h}$ (50\% higher if the experiment started in the early morning). If the calculation is extended to $12 \mathrm{~h}$, this effect is substantially reduced (ranging from $24 \%$ higher if it was started in early morning to $6 \%$ lower if it was started in late afternoon).

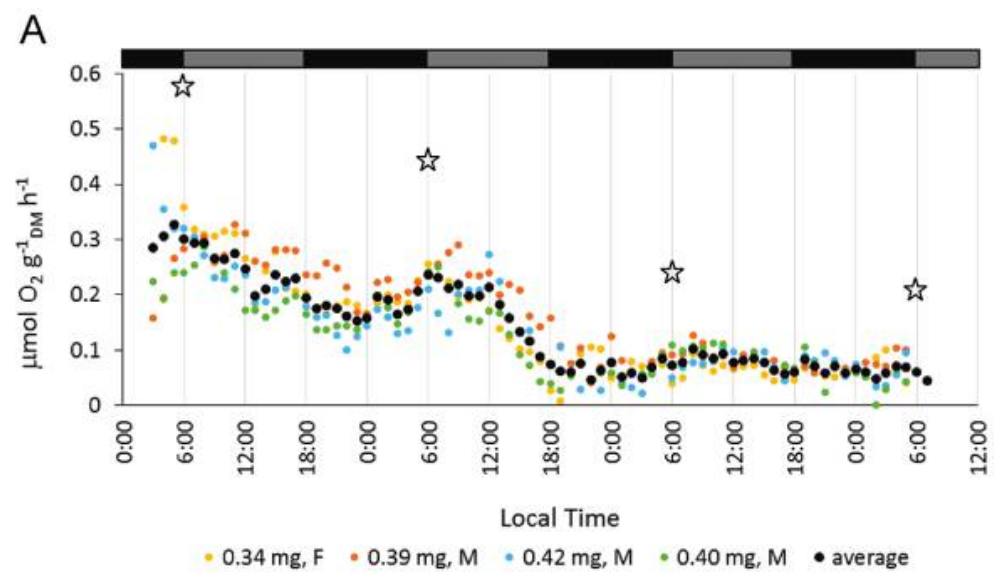

B

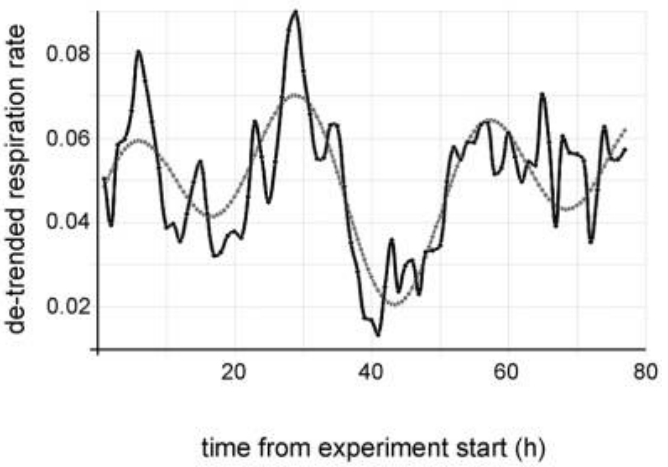

Figure 1. Circadian pattern in respiration rate. (A) Mass-specific respiration rate of 4 individuals maintained for 3 days in darkness at $20^{\circ} \mathrm{C}$. The dry mass and sex of each individual are noted in the legend. Periodicity was visualized by plotting 1-hour binned averages of the respiration rate $\left(\mu \mathrm{mol} \mathrm{O}_{2} \mathrm{~g}^{-1} \mathrm{DM}^{-1}\right)$. (B) Curve fit (gray line) to de-trended mean respiration rate (black line) using the fast Fourier transform non-linear least squares algorithm. 

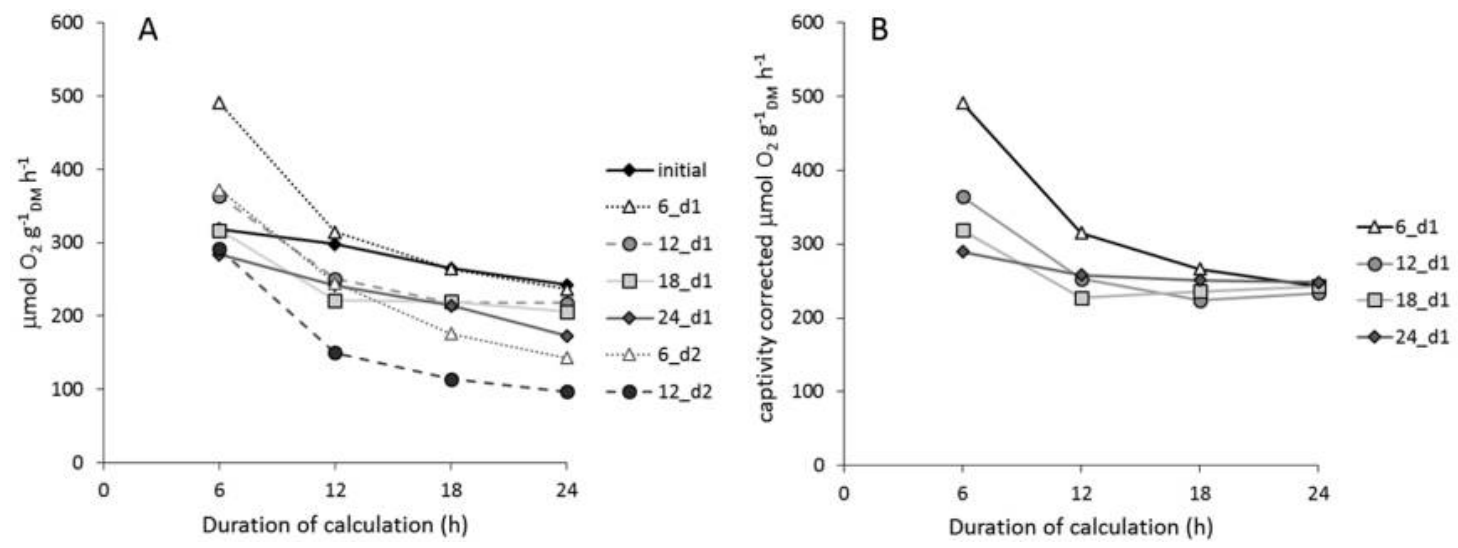

Figure 2. Effect of starting time and duration on rate calculation. The measured mass-specific metabolic rate (A) of Pleuromamma xiphias ( $y$-axis) is plotted based on how long the experiment was run ( $x$-axis) and using various starting points (the different lines correspond to the GMT time that the calculation was started). After correction for the decline in metabolic rate associated with captivity (B), it is obvious that the duration and time at which the experiment starts influence the calculated metabolic rate by up to $50 \%$.

Using these corrected values and the average dry mass (DM) of the copepods $(0.39 \mathrm{mg})$, we found a 24-h metabolic rate of $244 \mu \mathrm{mol} \mathrm{O}_{2} \mathrm{~g}^{-1}{ }_{\text {DM }} \mathrm{h}^{-1}$, with a daytime (06:00-18:00) rate of $315 \mu \mathrm{mol} \mathrm{O} \mathrm{g}^{-1} \mathrm{DM}^{-1}$ and a nighttime (18:00-06:00) rate of $220 \mu \mathrm{mol} \mathrm{O}_{2} \mathrm{~g}^{-1} \mathrm{DM} \mathrm{h}^{-1}$. Animals ranged in size from 2.47 to $3.37 \mathrm{mg}$ wet weight and from 0.34 to $0.42 \mathrm{mg}$ dry weight.

\section{Transcriptome}

The assembly metrics for the transcriptome were comparable to other recent pelagic crustacean de novo transcriptomes and sufficient to allow for mapping of protein identification. After quality trimming, the assembly was generated from 267 million paired-end reads. Of these, less than $0.4 \%$ were shorter than the 25 -bp cutoff that is Trinity's default setting for input. The final assembly, in which highly similar sequences were clustered via CD-HIT (Li and Godzik, 2006) with a 95\% similarity threshold, was composed of 554,991 Trinity transcripts and 458,857 Trinity "genes." Of the original trimmed reads, $94.08 \%$ were aligned back to this reference assembly, with $72.89 \%$ aligning concordantly as proper pairs. This very large assembly consists of several transcripts expressed at very low levels, most of which are likely to represent noncoding sequences, rare variants, and assembly artifacts. Only $22.7 \%$ are expressed at a level of at least 1 transcript per million. The average length of the transcripts was $445 \mathrm{bp}$, with a median length $308 \mathrm{bp}$ and an N50 of 469 bp but an E90N50 of 980. BUSCO analysis of the assembly reported a score of $89.3 \%$ completeness, with $16.2 \%$ duplication, $9.0 \%$ fragmentation, and $1.7 \%$ missing of 978 genes.

Annotation of the transcriptome with BLASTX versus nr (cutoff $1 \mathrm{e}^{-6}$ ) and Swiss-Prot (cutoff $1 \mathrm{e}^{-10}$ ), resulted in $22 \%$ and $16 \%$ annotation, respectively. Comparisons across other crustacean species using BLASTX at an e-value cutoff $1 \mathrm{e}^{-5}$ cross annotated anywhere between $14.5 \%$ (Euphausia superba; Meyer et al., 2015) and 30.1\% (Calanus finmarchicus; Tarrant et al., 2014) (Supplemental Data 2A, available online). The directed search for gene families of interest found matches for all previously identified genes that play roles in the major metabolic pathways, circadian rhythms, molting, and stress response for Crustacea (Christie et al., 2013; Meyer et al., 2015; Supplemental Data 2B, available online).

\section{Proteome}

Across all copepods analyzed, 1653 proteins were identified with high confidence in the proteomics data set (Supplemental Data 3, available online). Technical replicates showed a high degree of similarity, so NSAF values were averaged across replicates for the final analysis. Although there was no significant difference in full proteome profile between time points (ANOSIM, $R=0.193, P=0.076$; Fig. 3 ), there was a high similarity of protein abundance profiles for 3 individuals from the 20:00 time point, with $86 \%$ similarity compared to an average of $74 \%$ similarity among individuals (Fig. 3).

Of the 1653 proteins, $180(\sim 11 \%)$ were differentially abundant between the 2 time points with time-point-specific proteomic signatures. Ninety-seven of these proteins were detected at a higher abundance in copepods collected at 02:00 (positive logfold change), and 83 were higher in copepods collected at 20:00 (negative log-fold change). Distinct classes of proteins were represented in these differentially abundant sets (Supplemental Data 3, available online). At 20:00 P. xiphias was enriched for biological processes involving gas and/or oxygen transport and molecular function that GO terms "oxygen transporter activity," "iron ion binding," and "helicase activity." Proteins that were at significantly higher abundance in the 02:00 collected copepods were enriched for cellular components that included ribosome complexes and the molecular function "structural constituent of ribosome." 


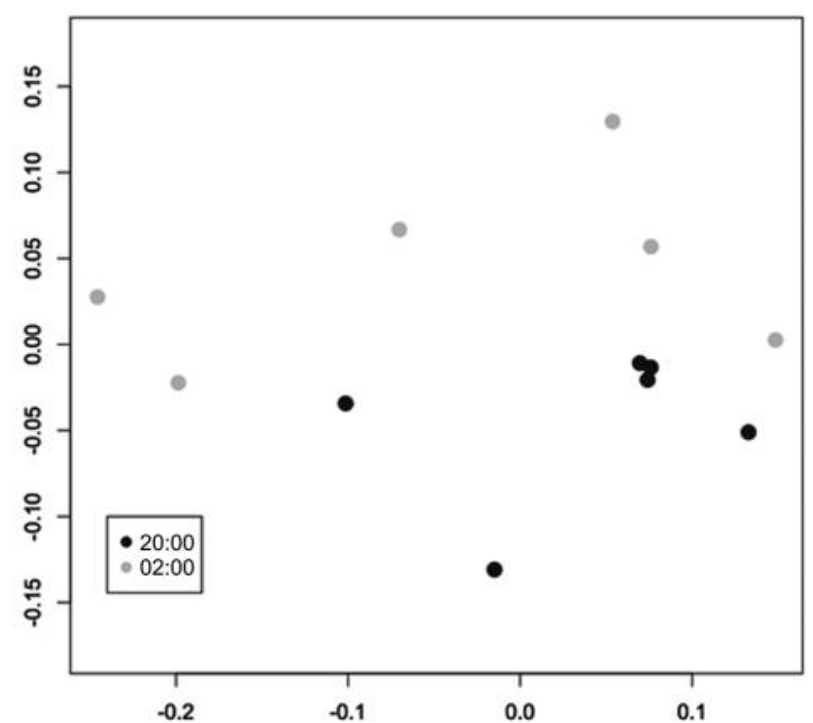

Figure 3. Non-metric multidimensional scaling (NMDS) plot of the abundance of complete proteome profile. Protein abundance values were $\log (x+1)$ transformed, and a Bray-Curtis dissimilarity matrix was constructed to compare individuals. An NMDS plot was built from this matrix, where each point corresponds to one adult female Pleuromamma xiphias. $2 \mathrm{D}$ stress $=0.08$.

At the level of individual proteins, there were several shifts in protein abundance that differentiated copepods from the two time points. Although differentially abundant proteins involved in transcription and translation and the cytoskeleton were present in both groups of copepods, they were much more prevalent in the surface-feeding animals at 02:00. DNA replication-related differentially abundant proteins were more prevalent in the upward migrators at 20:00 (7 vs. 2 differentially abundant proteins at 02:00). At each time point there were protein groups at higher abundance unique to that time point. At 20:00 the unique higher-abundance proteins were involved in the processes of lipid transport (2 proteins), bioluminescence (8 proteins), oxygen transport (4 proteins), shell matrix (1 protein), and hemolymph coagulation (1 protein). At 02:00 these were proteins involved in the muscle ( 2 proteins), lipid metabolism (4 proteins), nicotinamide adenine dinucleotide/reduced nicotinamide adenine dinucleotide (NAD/NADH) metabolism (4 proteins), and signal transduction (2 proteins). At both time points, proteins in a number of functional categories such as tricarboxylic acid (TCA) cycle proteins, adenosine triphosphate (ATP) binding and synthesis, carbohydrate metabolism, membrane proteins, calcium ion binding, reactive oxygen species response, and protein folding exhibited significant differential abundance.

\section{Discussion}

This study establishes a de novo transcriptome and proteome for one of the best-studied vertical migrators in the open ocean, Pleuromamma xiphias. These tools reveal changes in physiology between early evening, when the copepods first reach the surface (20:00), and early morning, when the copepods have been actively feeding in surface waters (02:00). While equipment constraints precluded sampling wild-caught organisms from the daytime portion of the diel cycle, our proteomic and transcriptomic resources indicate the feasibility and value of a larger-scale experiment that samples the organisms from their daytime deep-water environment. Respiration experiments suggest a true circadian pattern in metabolism, emphasizing the utility of this organism for exploring the interaction between migration and circadian rhythms in diel vertical migratory plankton and indicating that future molecular analyses should include mid-morning and mid-night sampling to better constrain this circadian signal.

\section{Respiration experiment}

Despite the continuous decline in respiration rate, which is likely a consequence of captivity and food deprivation, there was a strong and statistically significant cyclical pattern in oxygen consumption for the four respiration trials. The pattern, which showed peak metabolism in the early to mid-morning and a nadir at mid-night, persisted in the absence of a light cue, suggesting that it is a true circadian rhythm. Without a light signal to provide entrainment, circadian patterns can become attenuated over time. This is particularly evident in peripheral tissues studied in culture (reviewed in Hastings et al., 2003) but has also been described at the organismal level in diverse species, including copepods (Peres et al., 2014; Maas et al., 2016; Häfker et al., 2017).

This variation in metabolism has important implications for our current calculations of respiratory carbon demand, for zooplankton active flux via diel migration, and possibly for calculations of zooplankton contributions to nitrogen supply in the midwater. Biogeochemical calculations (Dam et al., 1995; Steinberg et al., 2000, 2002; Schnetzer and Steinberg, 2002b) typically do not document the time during which bottle experiments were started, and experiments often integrate metabolic rates over 6-24-h periods. For example, Teuber et al. (2013) reported an average respiration rate of 50 and $116 \mu \mathrm{mol}$ $\mathrm{O}_{2} \mathrm{~g}^{-1}{ }_{\text {DM }} \mathrm{h}^{-1}$ for adult (male and female, respectively) $P$. xiphias individuals from Cape Verde. These experiments were run 5-70 h after collection for a duration of 6-21 h and were performed with much larger individuals (0.87-1.82-mg males and 0.19-0.77-mg females compared with our 0.340.42-mg mixed gender). Steinberg et al. (2000) collected adult individuals (sex and individual dry mass undocumented) from the Sargasso Sea at night and ran experiments $\sim 8 \mathrm{~h}$ in duration, measuring a respiratory rate of $1.6 \mu \mathrm{g} \mathrm{C} \mathrm{mg}^{-1} \mathrm{DM} \mathrm{h}^{-1}$ at $24{ }^{\circ} \mathrm{C}$. Assuming a respiratory quotient of 0.87 (Mayzaud et al., 2005), this yields a respiration rate of $139 \mu \mathrm{mol}$ $\mathrm{O}_{2} \mathrm{~g}^{-1}{ }_{\mathrm{DM}} \mathrm{h}^{-1}$. The rates from both of these studies are less than half of the metabolic rate we measured for the captivitycorrected 12-h daytime (06:00-18:00) portion of our incuba- 
tion $\left(315 \mu \mathrm{mol} \mathrm{O} \mathrm{g}^{-1} \mathrm{DM} \mathrm{h}^{-1}\right)$. The respiration rate reported by Pavlova (1994) was an order of magnitude greater than that in our study, as well as those of Teuber et al. (2013) and Steinberg et al. (2000). Pavlova's (1994) highest respiration rate was $2500 \mu \mathrm{mol} \mathrm{O}_{2} \mathrm{~g}^{-1} \mathrm{DM}^{-1}$, with a daytime respiration rate of $312 \mu \mathrm{mol} \mathrm{O}_{2} \mathrm{~g}^{-1}{ }_{\mathrm{DM}} \mathrm{h}^{-1}$ and a similar dry weight as in the present study (0.29-0.39 mg).

Based on the results of our study, it is likely that an underestimation of the morning peak in the metabolic rate of $P$. $x i$ phias contributed to the lower metabolic rates reported by both Teuber et al. (2013) and Steinberg et al. (2000), although duration of the experiments and differences in organismal mass, food availability, and recent exposure to hypoxia (in the case of the Teuber et al. [2013] experiments) likely also played a role. The active flux of carbon to the deep ocean via migrator respiration only occurs during the daytime hours when organisms are at depth. Shorter respiration experiments starting in the early evening or those that extend a full $24 \mathrm{~h}$ would underestimate daytime respiration by $\sim 6 \%-24 \%$, based on our calculations. This is important because these reported respiratory rates are scaled to a community-level response and are used to estimate carbon flux based on an assumption that respiration is largely controlled by temperature, body mass, and oxygen availability (Ikeda, 2014; Kiko et al., 2016). Migrators of the genus Pleuromamma make up, on average, $\sim 10 \%$ of the nighttime biomass $(<200-\mu \mathrm{m}$ size fraction) at BATS, with the species P. xiphias making up $\sim 50 \%$ of the Pleuromamma biomass (Steinberg et al., 2000). Thus, our findings suggest that there is likely an appreciable underestimation of the calculation of active flux in the Sargasso Sea due to circadian variation in $P$. xiphias respiration rate. Should the circadian pattern in metabolism observed in our experiment be a broadscale pattern in plankton physiology, then our estimates of the biogeochemical contributions of DVM species to midwater processes during the daytime portion of their DVM would be substantially underestimated.

The consistency of the circadian pattern remains uncertain, however, because there now have been a number of variations reported in vertically migratory copepods. Recent work done with Calanus finmarchicus corroborates our findings that calanoid copepods have a true circadian pattern in metabolic rate (Häfker et al., 2017). The authors show a peak at 20:00 (dusk) and a nadir at 08:00 (3 h after dawn; mid-morning). It is difficult, however, to determine whether the patterns between these studies are similar, because the authors of the $C$. finmarchicus study plotted an anomaly of the decrease in oxygen rather than the respiration rate. Our findings are also somewhat different from the circadian pattern of diel vertically migratory copepods described by Pavlova (1994), who, during a study in the Indian Ocean between December and May, reported peaks in respiration about $2 \mathrm{~h}$ before sunrise (03:0005:00) and 4-6 h after sunset (22:00-24:00), with a nadir between 12:00 and 16:00. Pavlova (1994) also reports a similar pattern for Calanus euxinus collected in September 1992 in the Black Sea, with peaks at near sunrise (06:00) and $4 \mathrm{~h}$ after sunset (22:00) and a nadir at 12:00 and 02:00. Although these studies are difficult to compare, there does seem to be a consistent trend of increased respiration near dawn and dusk.

This trend is consistent with the idea that dawn and/or dusk metabolism would be the highest, as a consequence of the energetics associated with the migratory event. It is clear that DVM behavior is stimulated by light cues (reviewed in Cohen and Forward, 2009), and there is some evidence that DVM behavior is entrained by the light cycle and is under circadian control (Häfker et al., 2017). In our study, the presence of a peak only at dawn, with the dusk respiration being close to the lowest point of metabolic rate measured, adds complexity to this interpretation. Furthermore, the nighttime metabolic minimum observed in this study is inconsistent with current ideas of DVM physiology, which would predict higher evening metabolism. During the day, it is assumed that migratory organisms are in a quiescent phase, saving energy in the cold, dark depths, where food is scarce, while they are actively feeding and growing in the warm surface waters at night (McLaren, 1963; Loose and Dawidowicz, 1994). Pleuromamma xiphias is, however, thought to be one of the species of diel vertical migrators that actively feeds on particles at depth (Schnetzer and Steinberg, 2002a). Thus, an increased metabolic activity during the day at depth may be related to their particular ecology. Irrespective as to the cause, this nonconformity of circadian response across studies indicates that there is species-specific, regional, or seasonal variation. Understanding the speciesspecific and geographic patterns, along with their ecological drivers, will be important in refining our understanding of daytime respiration for calculations of biogeochemical flux.

\section{Transcriptome}

Although very little is known about copepod transcriptomes, much of the available information suggests that they can be very large. Recently sequenced calanoid copepod transcriptomes that use similar sequencing technology and assembly methods range in number of transcripts from $\sim 50,000$ to 240,000 (Supplemental Data 4, available online). Our assembly, which consists of more than 400,000 unique "genes," indicates that $P$. xiphias has a highly complex transcriptome in comparison to other calanoids. It is, however, very likely that many of the sequences are a consequence of short-read sequence-based assembly and the Trinity algorithms. Using analyses of transcript expression level (Ex) and associated weighted mean for a given expression level (ExN50), it is clear that sequences with extremely low abundance (between E99 and E100), which are difficult to assemble into long and meaningful contigs, make up $37 \%$ of the assembly. Choosing an Ex of only $98 \%$ puts the number of transcripts at $\sim 238,000$, while choosing an Ex of $90 \%$ reduces the number to $\sim 52,000$, well within the range of other assemblies. Despite the high number of rare transcripts, we feel that it is important to include all data in the assembly 
because the biological relevance of these contigs is impossible to determine. Importantly for the proteomics mapping, the assembly appears to be relatively complete, with our BUSCO score similar to that of the gene set of other more "model" invertebrates, including Caenorhabditis elegans and Lottia gigantea (Simão et al., 2015; Waterhouse et al., 2018), although there was a higher proportion of duplicated orthologs (16.2\% vs. $11.4 \%$ and $13.1 \%$, respectively). Pleuromamma belongs to the Augaptiliodea, the basal planktonic superfamily within the Calanoida (Blanco-Bercial et al., 2011; Bradford-Grieve et al., 2014). Most of the other available calanoid transcriptomes belong to Centropagoidea (e.g., Eurytemora) or Megacalanoidea (e.g., Calanus), both with very different ecological characteristics and evolutionary histories (Thuesen et al., 1998; Bradford-Grieve, 2002; Takenaka et al., 2012). To our knowledge, there is no other freely available annotated transcriptome for the Augaptiloidea to date, despite their prominence in open ocean environments and in the carbon and nitrogen export to the mesopelagic. This transcriptome thus adds a significantly different resource to the calanoid copepod genomic data available to the community.

Based on our analyses of transcripts expressed at low levels, it is likely that only about $23 \%$ of the transcripts were substantially expressed. Overall annotation via the nr and UniProt databases was somewhat limited, with a success rate of $20 \%$. The increased annotation success of the transcriptome when blasted against another copepod transcriptome (36\% vs. C. finmarchicus) provides reassurance that the likely coding regions are well assembled but reiterates that copepod transcriptomes are highly complex and require further research attention (Bron et al., 2011). The annotation of the assembly, which includes a directed search for homologs of known genes involved in molting, metabolic processes, stress response, and circadian rhythm, will allow for further analyses of these important processes.

\section{Proteome}

To our knowledge, this is the first study to annotate and measure more than a thousand proteins in a copepod proteome from single individuals, thus capturing inter-individual variability and highlighting the important proteomic physiological processes that are at play during surface residence time in these diel vertical migrators. Although there was no significant proteome-wide difference between the two time points, the differential abundance of copepod proteins revealed marked physiological signatures that were suggestive of changing metabolic processes, respiration, and antioxidant enzyme activity. It is important to note that individuals for the proteome study were collected only on one day for each time point, creating pseudoreplication in the experimental design. Furthermore, organisms were only sampled during the top portion of their diel migration and from two different days. These limitations make it difficult to attribute changing protein abundance ex- clusively to circadian cycling or migratory activity. The results do reveal significant changes in a subset of the protein classes that were hypothesized to be sensitive to these factors, and they validate the use of these tools to investigate diel changes in migrator physiology.

Most previous proteomic work with copepods has explored the stress responses of individuals based upon the proteomic responses of tens to hundreds of pooled individuals in laboratory experiments (Kimmel and Bradley, 2001; Zubrzycki et al., 2012; Boulangé-Lecomte et al., 2016), with only one other study taking a shotgun proteomics approach (Wang et al., 2017). The interpretation of these data is limited in ecological scope when in situ proteomes are uncharacterized and when inter-individual variability is masked by pooling. In contrast, the only proteomic studies on in situ copepod proteomes to date applied mass spectrometry methods with limited resolution (matrix-assisted laser desorption/ionization time-of-flight, MALDI-TOF) and limited interpretation for the purpose of species identification, rather than protein inference and physiology (Riccardi et al., 2012; Laakmann et al., 2013). Here, the characterization of individual proteomes from in situ samples reveals biologically and environmentally relevant physiological and metabolic patterns of an ecologically important species.

Although previous copepod studies do not report overall proteomic profiles to allow for direct comparison, the lack of overall proteomic differentiation is not surprising, as these proteomes were analyzed from wild-caught individuals that may vary in feeding history, reproductive state, precise age, and other factors. The number of proteins identified (1653) and the proportion that were differentially abundant $(\sim 11 \%$ of the proteome) were similar to what has been seen before when using a shotgun proteomics approach to study stress responses in other marine species. Wang et al. (2017) describe $\sim 3100$ proteins, $\sim 2 \%$ of which were differentially abundant in the proteome of the copepod Tigriopus japonicus exposed to acidification and/or mercury. In a study of oysters exposed to acidification and/or mechanical stress, Timmins-Shiffman et al. (2014) described 1616 proteins, $15 \%$ of which were differentially abundant.

The proteomes of the copepods that had recently arrived at the surface after migrating up from the depths (20:00 time point) showed evidence of increased oxygen transport, reproductive effort, and bioluminescence. Later in the evening (02:00) there was a higher abundance of a number of metabolic proteins, consistent with the carbohydrate, lipid, and general oxidative metabolism. A priori we hypothesized that we should see changes attributable to migration and circadian rhythmicity as variations in metabolic pathways, respiratory activity, antioxidant enzymes, and circadian proteins; and, thus, we focus the discussion on these patterns. With the exception of circadian proteins, there were significantly differentially abundant proteins associated with all of these functions in our analysis. Although sequences for circadian regulatory proteins were present in the transcriptome, most of these genes were expressed at 
low levels (Supplementary Data 4, available online), and none of them were detected within the proteome. Studies conducted in model animal systems demonstrate that expression of core regulatory transcripts, such as Clock and Period, are highest in neural "pacemaker" tissues, and relatively low levels are needed to regulate the expression of their downstream targets. Due to the high sensitivity of transcriptional profiling methods, this approach may provide better characterization of circadian physiology in future studies.

The increased abundance of four proteins involved in oxygen transport and iron binding at 20:00 may reflect the increased demand for internal oxygen transport to meet ATP demand during the upward migration of copepods through the water column. Three of these proteins were uncharacterized, and one was annotated as a metalloprotein when blasted against the Swiss-Prot database; all four, however, showed homology to hemerythrin, an invertebrate oxygen-binding protein with two iron atoms (reviewed in Stenkamp, 1994), when blasted against the NCBI nr protein database on May 26, 2017. A comparable increase in a blood oxygen-binding protein to support seasonal migration has been shown in birds, where increased concentration of red blood cells (hematocrit) and increased hemoglobin have been documented for migratory species and individuals preparing for or undergoing migration (Piersma et al., 1996; Landys-Ciannelli et al., 2002; Minias et al., 2013). Concurrently, 4 redox-associated proteins, including a protein disulfide-isomerase and a ubiquitin-activating enzyme, were detected at higher levels at 20:00, suggesting a signal of production of reactive oxygen species by these migrating individuals and the targeting of damaged proteins for degradation. Two other redox enzymes (dihydrolipoyl dehydrogenase and peroxiredoxin-6) were at increased abundance at the later 02:00 time point. Dihydrolipoyl dehydrogenase can have an antioxidant role by reducing ubiquinone to ubiquinol (Xia et al., 2001). As such, it may be a second wave of redox response, returning the organism to a reduced state after the stress of migration. Peroxiredoxins, in contrast, are well-known antioxidant enzymes that undergo circadian cycles in redox state that are deeply conserved across the tree of life and are sometimes accompanied by circadian cycles in the protein abundance (Edgar et al., 2012). Thus, although peroxiredoxin expression may be associated with an oxidative stress response, the variation in this protein may also be indicative of the natural circadian pattern of P. xiphias, and it is a good candidate for further exploration.

In the copepods that had been residing at the surface (collected at 02:00), there was proteomic evidence for increased digestive metabolism, where food is most abundant, with a higher abundance of carbohydrate metabolism proteins $(n=$ 7 proteins) and lipid metabolism proteins ( $n=4$ proteins). One of these proteins, the glycolytic enzyme glyceraldehyde3 -phosphate dehydrogenase, has previously been shown to have a diel cycle in dinoflagellates, with the enzyme abundance peaking in the late night and early day (Fagan et al.,
1999). More broadly, it is well established that there are circadian controls on many classes of genes associated with lipid metabolism and glucose homeostasis (Schlierf and Dorow, 1973; Marquez et al., 2004; Rudic et al., 2004). Thus, beyond changes associated with increased food availability in surface waters, this may be evidence of a circadian pattern in protein expression associated with metabolism in the late-evening copepods. Further analyses, controlling for the feeding state of the copepods and sampling over the diel cycle, will be required to determine whether light levels entrain these metabolic patterns independent of food availability.

Several proteins coding for enzymes directly involved in oxidative metabolism (NADH dehydrogenase, ATP synthase) were also at elevated levels at 02:00. They may similarly be linked to the circadian infrastructure associated with feeding. It has been demonstrated in mice that the circadian clock influences mitochondrial oxidative capacity via regulation of oxidized NAD $(\mathrm{NAD}+)$ biosynthesis and that these cofactors influenced lipid oxidation (Peek et al., 2013). The increase in these proteins may also be a precursor to the early-morning peak in metabolism documented in the respiration experiment.

\section{Conclusions}

Classical studies of migration detail pronounced changes in organismal physiology associated with long-duration, longdistance migrations in a suite of terrestrial and marine systems. In contrast, organisms that travel short distances, or those that consume food as they travel, often do not show pronounced physiological specialization associated with migration (Blem and Gauthreaux, 1980). Zooplankton migrations, which occur over a short period of time but over a very large distance, provide a distinctly different class of migration that has not been physiologically well studied to date. Furthermore, due to their reliance on daily light cues, these migrations are linked to circadian signaling. Although brief in duration, they are thought to require the expenditure of a large amount of energy in a short period of time, and it is unclear whether specific changes in physiology occur before, during, and/or after the migratory events. Disentangling the physiology of migration and circadian rhythm from the pronounced changes in the vertical environment, which involve transitions from the warm, well-oxygenated, food-replete surface to the cold, dark, food-poor midwater, has previously been difficult because of a lack of adequate molecular tools for assessment and less sensitive oxygen sensors.

This study, which provides a de novo transcriptome that acts as a template for proteomics analysis, is a useful first step toward approaching these questions. Our results suggest several pathways (e.g., respiratory oxygen-binding pigments, antioxidants, lipid, carbohydrate, and oxidative metabolism) that may respond to the migration event or be influenced by circadian signaling. With these tools in place, more substantial efforts, involving sampling over the full diel cycle at both surface and depth and/or sampling of cultured organisms under more 
controlled environmental conditions, will help to resolve underlying patterns of true circadian rhythmicity and the specific physiological changes that occur as these organisms make their daily migration. Understanding these circadian and migratory patterns is important, because, as shown by our respiration data, they can have an important and overlooked influence on our calculations of biogeochemical fluxes.

\section{Acknowledgments}

We would like to thank the captain and crew of the R/V Atlantic Explorer as well as the Bermuda Atlantic Time Series program (National Science Foundation grant OCE-1258622) for accommodating our opportunistic sampling of copepods. We are grateful to Steve Haddock for insights into interesting invertebrate physiology and Alex Federation for running test samples on the mass spectrometer. Michael Riffle created the gene ontology (GO) enrichment portal. We appreciate the insight provided by Sandra Spencer, Jason Gilmore, and Alyse Hawley on figures. Jimmy Eng gave indispensable advice on the proteomics searches and analysis. Brook Nunn assisted with the mass spectrometry analysis. Funding for ET-S was provided by a Training Grant from the National Institutes of Health (T32 HG00035), and proteomics work was supported in part by the University of Washington's Proteomics Resource (UWPR95794). Funding was provided by Simon's Foundation International as part of the BIOSSCOPE project.

\section{Data Accessibility}

Sequence and assembly data associated with this project are archived in GenBank under BioProject PRJNA352670. This Transcriptome Shotgun Assembly project has been deposited at DDBJ/EMBL/GenBank under the accession GFCI00000000. The version described in this paper is the first version, GFCI01000000.1. The mass spectrometry proteomics data have been deposited to the ProteomeXchange Consortium via the PRIDE partner repository with the data set identifier PXD005778.

\section{Literature Cited}

Al-Mutairi, H., and M. R. Landry. 2001. Active export of carbon and nitrogen at Station ALOHA by diel migrant zooplankton. Deep-Sea Res. II Top. Stud. Oceanogr. 48: 2083-2103.

Antezana, T. 2009. Species-specific patterns of diel migration into the Oxygen Minimum Zone by euphausiids in the Humboldt Current Ecosystem. Prog. Oceanogr. 83: 228-236.

Bautista, B., and R. Harris. 1992. Copepod gut contents, ingestion rates and grazing impact on phytoplankton in relation to size structure of zooplankton and phytoplankton during a spring bloom. Mar. Ecol. Prog. Ser. 82: 41-50.

Bianchi, D., and K. Mislan. 2016. Global patterns of diel vertical migration times and velocities from acoustic data. Limnol. Oceanogr. 61: 353-364.

BioDare2. 2016. BioDare2 beta. Fast period analysis, timeseries processing and aesthetic visualizations. [Online]. Available: https://biodare2.ed .ac.uk/ [2018, June 12].
Blanco-Bercial, L., and A. E. Maas. 2018. A transcriptomic resource for the northern krill Meganyctiphanes norvegica based on a short-term temperature exposure experiment. Mol. Ecol. Resour. 38: 25-32.

Blanco-Bercial, L., J. Bradford-Grieve, and A. Bucklin. 2011. Molecular phylogeny of the Calanoida (Crustacea: Copepoda). Mol. Phvlogenet. Evol. 59: 103-113.

Blem, C. R. 1980. The energetics of migration. Pp. 175-224 in Animal Migration, Orientation, and Navigation, S. A. Gauthreaux, Jr., ed. Academic Press, New York.

Bolger, A. M., M. Lohse, and B. Usadel. 2014. Trimmomatic: a flexible trimmer for Illumina sequence data. Bioinformatics 30: 2114-2120.

Boulangé-Lecomte, C., B. Rocher, K. Cailleaud, P. Cosette, E. Legrand, D. Devreker, H. Budzinski, S. Souissi, and J. Forget-Leray. 2016. Differential protein expression in the estuarine copepod Eurytemora affinis after diuron and alkylphenol exposures. Environ. Toxicol. Chem. 35: 1860-1871.

Bradford-Grieve, J. M. 2002. Colonization of the pelagic realm by calanoid copepods. Hydrobiologia 485: 223-244.

Bradford-Grieve, J. M., G. A. Boxshall, and L. Blanco-Bercial. 2014. Revision of basal calanoid copepod families, with a description of a new species and genus of Pseudocyclopidae. Zool. J Linn. Soc. 171: 507-533.

Bron, J. E., D. Frisch, E. Goetze, S. C. Johnson, C. E. Lee, and G. A. Wyngaard. 2011. Observing copepods through a genomic lens. Front. Zool. 8: 22.

Camacho, C., G. Coulouris, V. Avagyan, N. Ma, J. Papadopoulos, K. Bealer, and T. L. Madden. 2009. BLAST+: architecture and applications. BMC Bioinformatics 10: 421.

Childress, J. J., and B. A. Seibel. 1998. Life at stable low oxygen levels: adaptations of animals to oceanic oxygen minimum layers. J. Exp. Biol. 201: 1223-1232.

Choi, H., D. Fermin, and A. I. Nesvizhskii. 2008. Significance analysis of spectral count data in label-free shotgun proteomics. Mol. Cell. Proteom. 7: 2373-2385.

Christie, A. E., T. M. Fontanilla, K. T. Nesbit, and P. H. Lenz. 2013. Prediction of the protein components of a putative Calanus finmarchicus (Crustacea, Copepoda) circadian signaling system using a de novo assembled transcriptome. Comp. Biochem. Physiol. D Genom. Proteom. 8: 165-193.

Cohen, J. H., and R. B. Forward. 2009. Zooplankton diel vertical migration-a review of proximate control. Oceanogr. Mar. Biol. 47: 77109.

Dam, H. G., M. R. Roman, and M. J. Youngbluth. 1995. Downward export of respiratory carbon and dissolved inorganic nitrogen by dielmigrant mesozooplankton at the JGOFS Bermuda time-series station. Deep-Sea Res. I Oceanogr. Res. Pap. 42: 1187-1197.

De Pitta, C., A. Biscontin, A. Albiero, G. Sales, C. Millino, G. M. Mazzotta, C. Bertolucci, and R. Costa. 2013. The Antarctic krill Euphausia superba shows diurnal cycles of transcription under natural conditions. PLoS One 8: e68652.

Deutsch, E. W., L. Mendoza, D. Shteynberg, T. Farrah, H. Lam, N. Tasman, Z. Sun, E. Nilsson, B. Pratt, and B. Prazen. 2010. A guided tour of the Trans-Proteomic Pipeline. Proteomics 10: 1150-1159.

du Plessis, L., N. Škunca, and C. Dessimoz. 2011. The what, where, how and why of gene ontology - a primer for bioinformaticians. Brief. Bioinform. 12: 723-735.

Edgar, R. S., E. W. Green, Y. Zhao, G. van Ooijen, M. Olmedo, X. Qin, Y. Xu, M. Pan, U. K. Valekunja, and K. A. Feeney. 2012. Peroxiredoxins are conserved markers of circadian rhythms. Nature 485 : 459-464.

Eng, J. K., T. A. Jahan, and M. R. Hoopmann. 2013. Comet: an opensource MS/MS sequence database search tool. Proteomics 13: 22-24.

Eng, J. K., M. R. Hoopmann, T. A. Jahan, J. D. Egertson, W. S. Noble, and M. J. MacCoss. 2015. A deeper look into Comet-implementation and features. J. Am. Soc. Mass Spectrom. 26: 1865-1874. 
Enright, J., and W. Hamner. 1967. Vertical diurnal migration and endogenous rhythmicity. Science 157: 937-941.

Fagan, T., D. Morse, and J. W. Hastings. 1999. Circadian synthesis of a nuclear-encoded chloroplast glyceraldehyde-3-phosphate dehydrogenase in the dinoflagellate Gonyaulax polyedra is translationally controlled. Biochemistry 38: 7689-7695.

Fermin, D., V. Basrur, A. K. Yocum, and A. I. Nesvizhskii. 2011. Abacus: a computational tool for extracting and pre-processing spectral count data for label-free quantitative proteomic analysis. Proteomics 11: $1340-1345$.

Grabherr, M. G., B. J. Haas, M. Yassour, J. Z. Levin, D. A. Thompson, I. Amit, X. Adiconis, L. Fan, R. Raychowdhury, Q. D. Zeng, et al. 2011. Full-length transcriptome assembly from RNA-Seq data without a reference genome. Nat. Biotechnol. 29: 644-652.

Gwinner, E., and B. Helm. 2003. Circannual and circadian contributions to the timing of avian migration. Pp. 81-95 in Avian Migration, G. Zeichen, ed. Springer, Berlin.

Häfker, N. S., B. Meyer, K. S. Last, D. W. Pond, L. Hüppe, and M. Teschke. 2017. Circadian clock involvement in zooplankton diel vertical migration. Curr. Biol. 27: 2194-2201.

Hastings, M. H., A. B. Reddy, and E. S. Maywood. 2003. A clockwork web: circadian timing in brain and periphery, in health and disease. Nat. Rev. Neurosci. 4: 649 .

Hays, G. C. 2003. A review of the adaptive significance and ecosystem consequences of zooplankton diel vertical migrations. Hydrobiologia 503: $163-170$.

Heywood, K. J. 1996. Diel vertical migration of zooplankton in the Northeast Atlantic. J. Plankton Res. 18: 163-184.

Ikeda, T. 2014. Respiration and ammonia excretion by marine metazooplankton taxa: synthesis toward a global-bathymetric model. Mar. Biol. 161: 2753-2766.

Irigoien, X. 1998. Gut clearance rate constant, temperature and initial gut contents: a review. J. Plankton Res. 20: 997-1003.

Kiko, R., H. Hauss, F. Buchholz, and F. Melzner. 2016. Ammonium excretion and oxygen respiration of tropical copepods and euphausiids exposed to oxygen minimum zone conditions. Biogeosciences 13: 22412255.

Kimmel, D. G., and B. P. Bradley. 2001. Specific protein responses in the calanoid copepod Eurytemora affinis (Poppe, 1880) to salinity and temperature variation. J. Exp. Mar. Biol. Ecol. 266: 135-149.

Kobari, T., A. Shinada, and A. Tsuda. 2003. Functional roles of interzonal migrating mesozooplankton in the western subarctic Pacific. Prog. Oceanogr. 57: 279-298.

Kobari, T., D. K. Steinberg, A. Ueda, A. Tsuda, M. W. Silver, and M. Kitamura. 2008. Impacts of ontogenetically migrating copepods on downward carbon flux in the western subarctic Pacific Ocean. DeepSea Res. II Top. Stud. Oceanogr. 55: 1648-1660.

Laakmann, S., G. Gerdts, R. Erler, T. Knebelsberger, P. Martínez Arbizu, and M. Raupach. 2013. Comparison of molecular species identification for North Sea calanoid copepods (Crustacea) using proteome fingerprints and DNA sequences. Mol. Ecol. Resour. 13: 862-876.

Landys-Ciannelli, M. M., J. Jukema, and T. Piersma. 2002. Blood parameter changes during stopover in a long-distance migratory shorebird, the bar-tailed godwit Limosa lapponica taymyrensis. J. Avian Biol. 33: 451-455.

Li, W., and A. Godzik. 2006. CD-HIT: a fast program for clustering and comparing large sets of protein or nucleotide sequences. Bioinformatics 22: $1658-1659$.

Loose, C. J., and P. Dawidowicz. 1994. Trade-offs in diel vertical migration by zooplankton: the costs of predator avoidance. Ecology 75: 22552263.

Maas, A. E., I. T. Jones, A. M. Reitzel, and A. M. Tarrant. 2016. Daily cycle in oxygen consumption by the sea anemone Nematostella vectensis Stephenson. Biol. Open 5: 161-164.
Marquez, S., P. Crespo, V. Carlini, E. Garbarino-Pico, R. Baler, B. L. Caputto, and M. E. Guido. 2004. The metabolism of phospholipids oscillates rhythmically in cultures of fibroblasts and is regulated by the clock protein PERIOD 1. FASEB J. 18: 519-521.

Mauchline, J. 1998. The Biology of Calanoid Copepods. Academic Press, London.

Mayzaud, P., M. Boutoute, S. Gasparini, L. Mousseau, and D. Lefevre. 2005. Respiration in marine zooplankton - the other side of the coin: $\mathrm{CO}_{2}$. Limnol. Oceanogr. 50: 291-298.

McGuire, L. P., and C. G. Guglielmo. 2009. What can birds tell us about the migration physiology of bats? J. Mammal. 90: 1290-1297.

McLaren, I. A. 1963. Effects of temperature on growth of zooplankton, and the adaptive value of vertical migration. J. Fish. Res. Board Can. 20: 685-727.

Meyer, B., P. Martini, A. Biscontin, C. De Pittà, C. Romualdi, M. Teschke, S. Frickenhaus, L. Harms, U. Freier, and S. Jarman. 2015. Pyrosequencing and de novo assembly of Antarctic krill (Euphausia superba) transcriptome to study the adaptability of krill to climateinduced environmental changes. Mol. Ecol. Resour. 15: 1460-1471.

Minias, P., K. Kaczmarek, R. Włodarczyk, and T. Janiszewski. 2013. Hemoglobin concentrations in waders vary with their strategies of migration: a comparative analysis. Comp. Biochem. Physiol. A Mol. Integr. Physiol. 165: 7-12.

Oksanen, J., F. Blanchet, R. Kindt, P. Legendre, P. Minchin, R. O'Hara, G. Simpson, P. Solymos, M. Stevens, and H. Wagner. 2016. vegan: community ecology package, version 2.4-1. [Online]. Available: https:// CRAN.R-project.org/package = vegan [2018, July 16].

Pavlova, E. 1994. Diel changes in copepod respiration rates. Hydrobiologia 292: 333-339.

Peek, C. B., A. H. Affinati, K. M. Ramsey, H.-Y. Kuo, W. Yu, L. A. Sena, O. Ilkayeva, B. Marcheva, Y. Kobayashi, and C. Omura. 2013. Circadian clock NAD + cycle drives mitochondrial oxidative metabolism in mice. Science 342: 1243417.

Peres, R., A. M. Reitzel, Y. Passamaneck, S. C. Afeche, J. Cipolla-Neto, A. C. Marques, and M. Q. Martindale. 2014. Developmental and light-entrained expression of melatonin and its relationship to the circadian clock in the sea anemone Nematostella vectensis. EvoDevo 5: 26.

Piersma, T., J. M. Everaarts, and J. Jukema. 1996. Build-up of red blood cells in refuelling bar-tailed godwits in relation to individual migratory quality. Condor 98: 363-370.

R Core Team. 2016. R: a language and environment for statistical computing. [Online]. R Foundation for Statistical Computing, Vienna. Available: http://www.R-project.org [2018, July 16].

Riccardi, N., L. Lucini, C. Benagli, M. Welker, B. Wicht, and M. Tonolla. 2012. Potential of matrix-assisted laser desorption/ionization time-of-flight mass spectrometry (MALDI-TOF MS) for the identification of freshwater zooplankton: a pilot study with three Eudiaptomus (Copepoda: Diaptomidae) species. J. Plankton Res. 34: 484-492.

Rudic, R. D., P. McNamara, A.-M. Curtis, R. C. Boston, S. Panda, J. B. Hogenesch, and G. A. FitzGerald. 2004. BMAL1 and CLOCK, two essential components of the circadian clock, are involved in glucose homeostasis. PLoS Biol. 2: e377.

Schlierf, G., and E. Dorow. 1973. Diurnal patterns of triglycerides, free fatty acids, blood sugar, and insulin during carbohydrate-induction in man and their modification by nocturnal suppression of lipolysis. J. Clin. Invest. 52: 732.

Schnetzer, A., and D. Steinberg. 2002a. Natural diets of vertically migrating zooplankton in the Sargasso Sea. Mar. Biol. 141: 89-99.

Schnetzer, A., and D. K. Steinberg. 2002b. Active transport of particulate organic carbon and nitrogen by vertically migrating zooplankton in the Sargasso Sea. Mar. Ecol. Prog. Ser. 234: 71-84.

Simão, F. A., R. M. Waterhouse, P. Ioannidis, E. V. Kriventseva, and E. M. Zdobnov. 2015. BUSCO: assessing genome assembly and annotation completeness with single-copy orthologs. Bioinformatics 31: 3210-3212. 
Skrip, M. M., and S. R. McWilliams. 2016. Oxidative balance in birds: an atoms-to-organisms-to-ecology primer for ornithologists. J. Field Ornithol. 87: 1-20.

Steinberg, D. K., and M. R. Landry. 2017. Zooplankton and the Ocean Carbon Cycle. Annu. Rev. Mar. Sci. 9: 413-444.

Steinberg, D. K., C. A. Carlson, N. R. Bates, S. A. Goldthwait, L. P. Madin, and A. F. Michaels. 2000. Zooplankton vertical migration and the active transport of dissolved organic and inorganic carbon in the Sargasso Sea. Deep-Sea Res. I Oceanogr. Res. Pap. 47: 137-158.

Steinberg, D. K., S. A. Goldthwait, and D. A. Hansell. 2002. Zooplankton vertical migration and the active transport of dissolved organic and inorganic nitrogen in the Sargasso Sea. Deep-Sea Res. I Oceanogr. Res. Pap. 49: 1445-1461.

Steinberg, D. K., J. S. Cope, S. E. Wilson, and T. Kobari. 2008. A comparison of mesopelagic mesozooplankton community structure in the subtropical and subarctic North Pacific Ocean. Deep-Sea Res. II Top. Stud. Oceanogr. 55: 1615-1635.

Stenkamp, R. E. 1994. Dioxygen and hemerythrin. Chem. Rev. 94: 715726.

Takenaka, Y., A. Yamaguchi, N. Tsuruoka, M. Torimura, T. Gojobori, and Y. Shigeri. 2012. Evolution of bioluminescence in marine planktonic copepods. Mol. Biol. Evol. 29: 1669-1681.

Tarrant, A. M., M. F. Baumgartner, B. H. Hansen, D. Altin, T. Nordtug, and A. J. Olsen. 2014. Transcriptional profiling of reproductive development, lipid storage and molting throughout the last juvenile stage of the marine copepod Calanus finmarchicus. Front. Zool. 11: 1.

Teschke, M., S. Wendt, S. Kawaguchi, A. Kramer, and B. Meyer. 2011. A circadian clock in Antarctic krill: an endogenous timing system governs metabolic output rhythms in the euphausid species Euphausia superba. PLoS One 6: e26090.

Teuber, L., R. Kiko, F. Séguin, and H. Auel. 2013. Respiration rates of tropical Atlantic copepods in relation to the oxygen minimum zone. $J$. Exp. Mar. Biol. Ecol. 448: 28-36.

Thaben, P. F., and P. O. Westermark. 2014. Detecting rhythms in time series with RAIN. J. Biol. Rhvthms 29: 391-400.

Thuesen, E. V., C. B. Miller, and J. J. Childress. 1998. Ecophysiological interpretation of oxygen consumption rates and enzymatic activities of deep-sea copepods. Mar. Ecol. Prog. Ser. 168: 95-107.
Timmins-Schiffman, E., B. L. Nunn, D. R. Goodlett, and S. B. Roberts. 2013. Shotgun proteomics as a viable approach for biological discovery in the Pacific oyster. Conserv. Physiol. 1: cot009.

Timmins-Schiffman, E., W. D. Coffey, W. Hua, B. L. Nunn, G. H. Dickinson, and S. B. Roberts. 2014. Shotgun proteomics reveals physiological response to ocean acidification in Crassostrea gigas. mics 15: 951.

Timmins-Schiffman, E. B., G. A. Crandall, B. Vadopalas, M. E. Riffle, B. L. Nunn, and S. B. Roberts. 2017. Integrating discovery-driven proteomics and selected reaction monitoring to develop a noninvasive assay for geoduck reproductive maturation. J. Proteome Res. 16: 3298-3309.

UniProt Consortium. 2016. UniProt: the universal protein resource. [Online]. Available: https://www.uniprot.org [2016, September 29].

Wang, M., J.-S. Lee, and Y. Li. 2017. Global proteome profiling of a marine copepod and the mitigating effect of ocean acidification on mercury toxicity after multigenerational exposure. Environ. Sci. Technol. 51: 5820-5831.

Waterhouse, R. M., M. Seppey, F. A. Simão, M. Manni, P. Ioannidis, G. Klioutchnikov, E. V. Kriventseva, and E. M. Zdobnov. 2018. BUSCO applications from quality assessments to gene prediction and phylogenomics. Mol. Biol. Evol. 35: 543-548.

Wiebe, P. H., N. J. Copley, and S. H. Boyd. 1992. Coarse-scale horizontal patchiness and vertical migration of zooplankton in Gulf Stream warm-core ring 82-H. Deep-Sea Res. I Oceanogr. Res. Pap. 39 (suppl.): S247-S278.

Wormuth, J. 1981. Vertical distributions and diel migrations of Euthecosomata in the northwest Sargasso Sea. Deep-Sea Res. I Oceanogr. Res. Pap. 28: 1493-1515.

Xia, L., M. Björnstedt, T. Nordman, L. C. Eriksson, and J. M. Olsson. 2001. Reduction of ubiquinone by lipoamide dehydrogenase. FEBS J. 268: $1486-1490$

Zielinski, T., A. M. Moore, E. Troup, K. J. Halliday, and A. J. Millar. 2014. Strengths and limitations of period estimation methods for circadian data. PLoS One 9: e96462.

Zubrzycki, I. Z., S. Lee, K. Lee, M. Wiacek, and W. Lee. 2012. The study on highly expressed proteins as a function of an elevated ultraviolet radiation in the copepod, Tigriopus japonicus. Ocean Sci. J. 47: 75-82. 